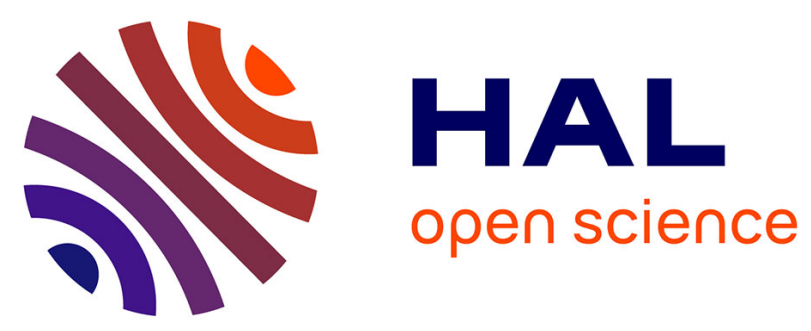

\title{
Identification of surface and volume hotcarrier thermalization mechanisms in ultrathin GaAs layers
}

Maxime Giteau, Edouard de Moustier, Daniel Suchet, Hamidreza

Esmaielpour, Hassanet Sodabanlu, Kentaroh Watanabe, Stéphane Collin, Jean-François Guillemoles, Yoshitaka Okada

\section{To cite this version:}

Maxime Giteau, Edouard de Moustier, Daniel Suchet, Hamidreza Esmaielpour, Hassanet Sodabanlu, et al.. Identification of surface and volume hotcarrier thermalization mechanisms in ultrathin GaAs layers. Journal of Applied Physics, 2020, 128 (19), pp.193102. 10.1063/5.0027687 . hal-03022819

\section{HAL Id: hal-03022819 https://hal.science/hal-03022819}

Submitted on 24 Nov 2020

HAL is a multi-disciplinary open access archive for the deposit and dissemination of scientific research documents, whether they are published or not. The documents may come from teaching and research institutions in France or abroad, or from public or private research centers.
L'archive ouverte pluridisciplinaire HAL, est destinée au dépôt et à la diffusion de documents scientifiques de niveau recherche, publiés ou non, émanant des établissements d'enseignement et de recherche français ou étrangers, des laboratoires publics ou privés. 


\title{
Identification of surface and volume hot- carrier thermalization mechanisms in ultrathin GaAs layers
}

\author{
Maxime Giteau ${ }^{1,4,}{ }^{*}$, Edouard de Moustier ${ }^{1,4}$, Daniel Suchet ${ }^{2,4}$, Hamidreza Esmaielpour ${ }^{2,4}$, \\ Hassanet Sodabanlu ${ }^{1,4}$, Kentaroh Watanabe ${ }^{1,4}$, Stéphane Collin ${ }^{3,4}$, \\ Jean-François Guillemoles ${ }^{2,4,}{ }^{*}$, Yoshitaka Okada ${ }^{1,4}$
}

${ }^{1}$ Research Center for Advanced Science and Technology, The University of Tokyo, Komaba 4-6-1, Meguro-ku, Tokyo 153-8904, Japan.

${ }^{2}$ CNRS, Ecole Polytechnique, Institut Photovoltaique d'lle de France (IPVF), UMR 9006, 18 Boulevard Thomas Gobert, 91120 Palaiseau, France.

${ }^{3}$ Centre de Nanosciences et de Nanotechnologies (C2N), CNRS, Université Paris-Saclay, 10 Boulevard Thomas Gobert, 91120 Palaiseau, France.

${ }^{4}$ NextPV, LIA RCAST-CNRS, The University of Tokyo, Komaba 4-6-1, Meguro-ku, Tokyo 1538904, Japan.

*Corresponding authors: maximegiteau@hotmail.fr; jf.guillemoles@cnrs.fr

\begin{abstract}
Hot-carrier solar cells offer the opportunity to harvest more energy than the limit set by the ShockleyQueisser model by reducing the losses due to the thermalization of photo-generated carriers. Previous reports have shown lower thermalization rates in thinner absorbers, but the origin of this phenomenon is not precisely understood. In this work, we investigate a series of ultrathin GaAs absorber layers sandwiched between AlGaAs barriers and transferred on host substrates with a gold back mirror. We perform power-dependent photoluminescence characterizations at different laser wavelengths from which we determine the carrier temperature in four absorber thicknesses between 20 and $200 \mathrm{~nm}$. We observe a linear relationship between the absorbed power and the carrier temperature increase. By relating the absorbed and thermalized power, we extract a thermalization coefficient for all samples. It shows an affine dependence with the thickness, leading to the identification of distinct volume and surface contributions to thermalization. We confirm that volume thermalization is linked to LO phonon decay. We discuss the origin of the interface-related thermalization, showing that the effect of LO phonon transport is negligible. Overall, this work sheds new light on thermalization processes in ultrathin semiconductor layers and introduces a method to compare the performance of hot-carrier absorbers.
\end{abstract}

\section{INTRODUCTION}

Conventional single-junction solar cells are limited in efficiency by the Shockley-Queisser model $^{1}$, at about $41 \%$ under full concentration for an optimal bandgap. This efficiency limit comes from the mismatch between a broadband solar illumination, and the fixed bandgap of the semiconductor used to absorb this solar radiation. Photons with an energy lower than the bandgap cannot be absorbed, while high-energy photons can be absorbed, but all the energy above the bandgap is lost through so-called 
thermalization. Hot-carrier solar cells (HCSCs), with an efficiency limit around $85 \%$ under full concentration ${ }^{2}$, offer the opportunity to solve this issue. Still, to date, only a few complete devices have been fabricated ${ }^{3-5}$. Among these, the most convincing example showed a maximal efficiency of $11 \%$ at room temperature ${ }^{4}$, far below the limit set by the Shockley-Queisser model.

Two specific elements are required for a HCSC. The first is an absorber with a low thermalization rate, resulting in electrons and holes at a higher temperature than the lattice. The second is energy-selective contacts ${ }^{6-8}$, which allow for the isentropic extraction of these hot carriers. Although understanding the selective contacts is crucial for reaching the highest efficiencies, a simple barrier implemented as a semi-selective contact could already give a substantial efficiency increase from hot carriers ${ }^{9}$. Therefore, the main challenge lies in the ability to reduce the thermalization rate in the absorber and generate a steady-state hot-carrier population.

In bulk polar materials such as GaAs, the excess energy of electrons is mostly released as LO phonons through elastic scattering known as the Fröhlich interaction ${ }^{10}$. Electrons can also re-absorb LO phonons, meaning the electron-LO phonon system conserves energy. The energy loss then comes from the anharmonic decay of LO phonons into acoustic phonons ${ }^{11}$ (mainly through Klemens and Ridley mechanisms). The result of these interactions creates a nonequilibrium LO phonon distribution ${ }^{12,13}$.

When a thin absorber is sandwiched between two barrier layers, thermalization can also occur through the interfaces. One such mechanism is thermionic emission, where carriers with more energy than the barrier height can escape from the absorber. This mechanism is employed for thermionic cooling applications, where the carrier temperature in the absorber is lowered by extracting highenergy carriers ${ }^{14,15}$. Although it is sometimes dominant in hot-carrier absorbers ${ }^{16}$, its effect can be mitigated by using barriers with a bandgap much larger than the absorber. Another mechanism thought to be of influence is the transmission of LO phonons through the interfaces, which can be reduced by using absorber and barrier materials with different LO phonon energies (like GaAs and AIAs) ${ }^{17}$.

Initial studies have shown that the thermalization rate was strongly reduced in quantum wells compared to bulk absorbers ${ }^{18,19}$, while recent works have focused on the mechanisms occurring in complex multiple quantum well structures ${ }^{20-22}$. Despite these advances, the reason why the thermalization rate is lower in quantum systems is still debated.

Although quantum confinement certainly plays a role, part of this reduction can purely be ascribed to having a thinner absorber. This is because the number of LO phonon decay channels scales with the absorber volume ${ }^{23}$. Other identified thermalization mechanisms include Auger effect ${ }^{24}$, although it is significant only for carrier concentrations above $10^{19} \mathrm{~cm}^{-3}$ in $\mathrm{GaAs}^{25}$.

In this work, we investigate the impact of the absorber thickness on thermalization in a range where the absorbers behave like bulk materials (no quantum confinement). We fabricate simple barrier-absorber-barrier heterostructures with GaAs absorbers of different thicknesses and compare the power-dependent photoluminescence measurements for each sample to identify the main thermalization mechanisms in thin absorbers.

In section II, we introduce ultrathin heterostructures transferred on a host substrate with 4 different GaAs absorber thicknesses between 20 and $200 \mathrm{~nm}$. They are characterized by room-temperature photoluminescence $(\mathrm{PL})$ with two different laser wavelengths of variable intensity. We present a method to extract the carrier temperature in ultrathin devices with optical resonances.

In section III, we present the temperatures determined for all samples at different laser wavelengths and intensities. We observe a linear relationship between the absorbed power and the carrier temperature increase so that we can extract a proportionality coefficient for all samples.

In section IV, we discuss the experimental results. We relate the proportionality coefficient to a thermalization coefficient $Q$, by defining a relationship between absorbed and thermalized power. By comparing the thermalization coefficient obtained for different thicknesses, we identify separate volume and surface contributions to thermalization. The volume thermalization seems to correspond to the LO phonon decay mechanism, while we exclude the transmission of LO phonons through the barriers as a possible surface thermalization mechanism.

In section $\mathrm{V}$, we conclude on the main results of this study and their perspectives to enhance hot-carrier phenomena in solar cells.

\section{METHODS}

We fabricated four samples, with GaAs absorbers of thicknesses 20, 50, 100, and $200 \mathrm{~nm}$ (Figure 1(a)). The samples are epitaxially grown by metalorganic vapor-phase epitaxy, then bonded to a silicon wafer through gold-gold bonding. Finally, the growth substrate is removed by chemical etching. The whole fabrication process, from the epitaxial growth on GaAs to the final device, has been reported previously ${ }^{26}$. For each sample, the absorber layer is sandwiched between 2 wider-bandgap $\mathrm{Al}_{0.5} \mathrm{Ga}_{0.5} \mathrm{As}$ barriers of about $90 \mathrm{~nm}$ thickness (Figure 1(b)). The purpose 
of these barriers is to confine the carriers in a well-defined volume and limit the power outflow from the absorber through thermionic emission. The exact thickness of the barriers can vary due to partial etching and oxidation during the process (we determined it previously for each sample ${ }^{26}$ ). The gold layer used for the bonding also plays the role of a mirror and ensures high thermal conductivity. This design makes the sample as simple as possible to analyze with PL. Indeed, since there is no substrate and the barriers have a wide bandgap, only the absorber will emit light in the spectral region of interest (between 1.35 and $1.6 \mathrm{eV}$ ). Also, minimizing lattice heating leads to a more accurate measurement of the carrier temperature, since we can assume the lattice remains at room temperature even under intense illumination.

(a)
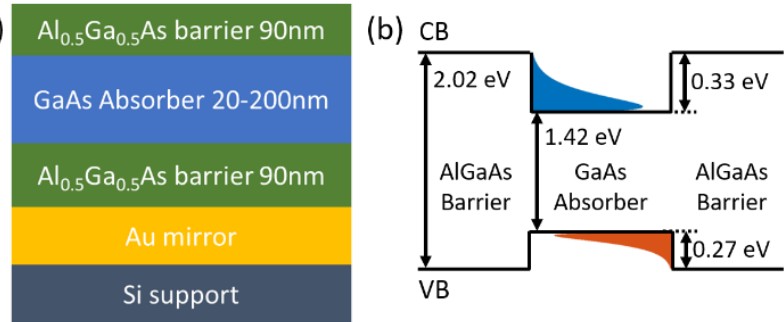

Figure 1: (a) Structure of the samples measured in this study. A GaAs absorber is sandwiched between two $\mathrm{Al}_{0.5} \mathrm{Ga}_{0.5} \mathrm{As}$ barriers with a higher bandgap. The structure is bonded to a silicon substrate with a gold layer to limit lattice heating. (b) Simplified band diagram of the barrier-absorber-barrier structure, along with the bandgaps and band offsets. The electron and hole energy distributions are represented in blue and red, respectively. The vast majority of the carriers in the absorber do not have enough energy to escape to the barriers through thermionic emission.

To compare the measurements for different absorber thicknesses, we need to consider the fraction $P_{a b s}$ (in W. $\mathrm{cm}^{-2}$ ) of the incident laser power $P_{\text {inc }}$ (in W. $\mathrm{cm}^{-2}$ ) which is absorbed in each of them:

$$
P_{\text {abs }}=A_{\text {laser }} \times P_{\text {inc }}
$$

where $A_{\text {laser }}$ is the absorptivity of the GaAs layer at the laser wavelength. $A_{\text {laser }}$ was determined previously ${ }^{26}$ for each sample by fitting absorptivity measurements with a transfer matrix method ${ }^{27,28}$. We consider $A_{\text {laser }}$ is independent of the incident power, because the laser energies considered are far above the bandgap.

To determine the carrier temperature in the absorber as a function of the absorbed power, we measure the PL spectra at room temperature under laser illumination of increasing intensity in the four samples. We consider two laser wavelengths of 532 and $638 \mathrm{~nm}$ corresponding to photon energies of 2.33 and $1.94 \mathrm{eV}$, respectively. These energies are significantly higher than the absorber bandgap $(1.42 \mathrm{eV})$, such that we can expect a significant part of the incident power to be thermalized. The photons emitted by the $638 \mathrm{~nm}$ laser have less energy than the barrier bandgap

$(2.02 \mathrm{eV})$. Photocarriers are thus generated only in the absorber, which is not the case for the $532 \mathrm{~nm}$ laser (more detail on how this impacts the estimation of the absorbed power in Supplementary Information). Thanks to these two different lasers, we will be able to analyze the wavelength

dependence of thermalization. The series of spectra obtained for the $200 \mathrm{~nm}$-thick GaAs absorber with the 532 $\mathrm{nm}$ laser is shown in Figure 2.

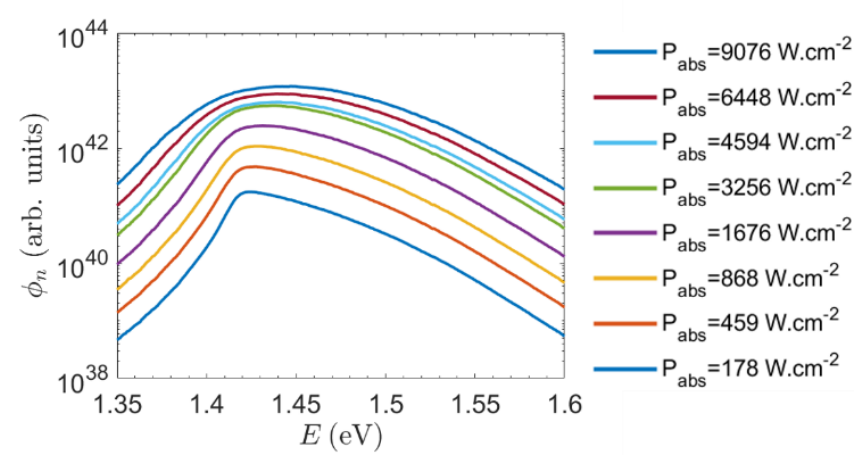

Figure 2: PL spectral photon flux (in arbitrary units) as a function of the photon energy, acquired at different absorbed powers for the $200 \mathrm{~nm}$-thick GaAs absorber sample with a $532 \mathrm{~nm}$ illumination. The laser spot size is about $19 \mu \mathrm{m}$ in radius (see Supplementary Information).

The PL spectral photon flux (in $\mathrm{s}^{-1} \cdot \mathrm{m}^{-2} \cdot \mathrm{eV}^{-1}$ ) of a semiconductor with a Lambertian emission profile follows the generalized Planck law ${ }^{29}$

$$
\phi(E)=A(E) \frac{2 E^{2}}{h^{3} c^{2}}\left[\exp \left(\frac{E-\mu}{k_{B} T}\right)-1\right]^{-1},
$$

where $E$ is the photon energy, $A(E)$ is the absorptivity of the GaAs layer (considered independent of the emission angle), $h$ is the Planck constant, $c$ is the speed of light, $k_{B}$ is the Boltzmann constant, $\mu$ is the chemical potential of the radiation and $T$ is the temperature of the radiation. In the Boltzmann approximation, Eq. (1) becomes

$$
\phi(E) \approx K A(E) E^{2} \exp \left(-\frac{E}{k_{B} T}\right),
$$

where $K$ is a parameter independent of the photon energy (it depends on the absorber thickness and illumination conditions but has no impact on the carrier temperature determination). If electrons and holes are at the same 
temperature, then $T$ is also the temperature of the carriers. If electrons and holes are at different temperatures, Eq. (2) is still valid, with $T$ related to the temperature of the carrier populations $\mathrm{s}^{30,31}$. We will consider here, as is usually assumed, that the carrier temperatures are the same. The same analysis could be conducted for different electron and hole temperatures.

A simple way to extract the carrier temperature $T$ from $\mathrm{PL}$ measurements is the slope method ${ }^{20}$. This method assumes the absorptivity is independent of $E$ on a certain range above the bandgap to extract the temperature directly from the slope of the PL spectrum in logarithmic scale in that range. However, in the ultrathin systems considered here, Fabry-Perot resonances appear between the front interface and the back mirror ${ }^{26}$. These resonances result in strong absorption variations that can lead to significant errors in the carrier temperature assessment.

We have proposed an alternative method to extract the temperature in thin heterostructures ${ }^{32}$, where we consider the ratio between the PL spectra obtained at two different laser fluences labeled 1 and $n$. Assuming the absorptivity at a given energy is independent of the absorbed power, we have

(a) $10^{3}$

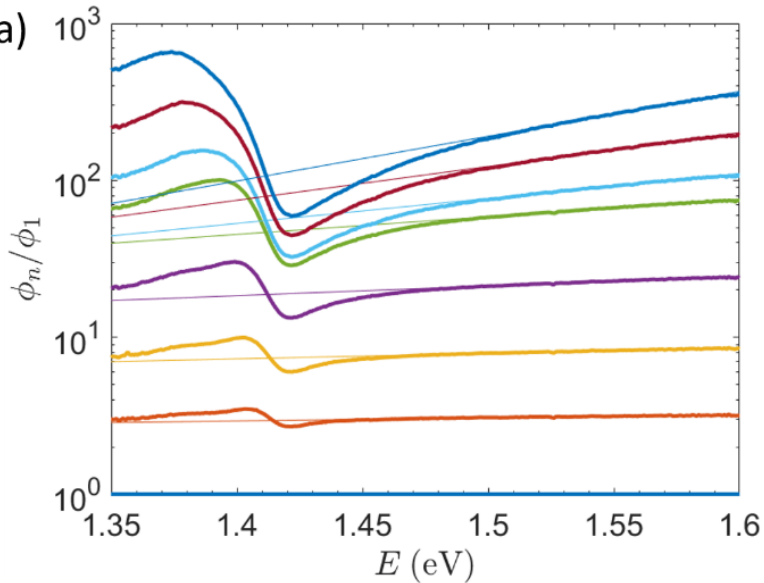

$$
\ln \left(\frac{\phi_{n}(E)}{\phi_{1}(E)}\right)=\left(\frac{1}{k_{B} T_{1}}-\frac{1}{k_{B} T_{n}}\right) E+\text { cste. }
$$

The high-energy slope of this ratio gives directly the temperature of the carriers. This approach is as simple to implement as the slope method, but presents the extra advantage of removing the influence of the absorption variations with $E$. Also, both these methods are much simpler to apply than a full fitting ${ }^{4}$ of Eq. (2) since there is no need for an absolute calibration of the photoluminescence intensity, and there is only one parameter to fit.

The PL ratios are shown in Figure 3(a), for the PL spectra of the $200 \mathrm{~nm}$-thick GaAs absorber presented in Figure 2. The temperature determination is about twice more accurate with the ratio method than with the slope method for the $200 \mathrm{~nm}$ absorber case (Figure 3(b)).

This method can be used as long as $A(E)$ is independent of the absorbed power. Close to the bandgap, intensitydependent band filling occurs, which means the temperature needs to be determined sufficiently far above the bandgap (in our case, above $1.53 \mathrm{eV}$ ). (b)

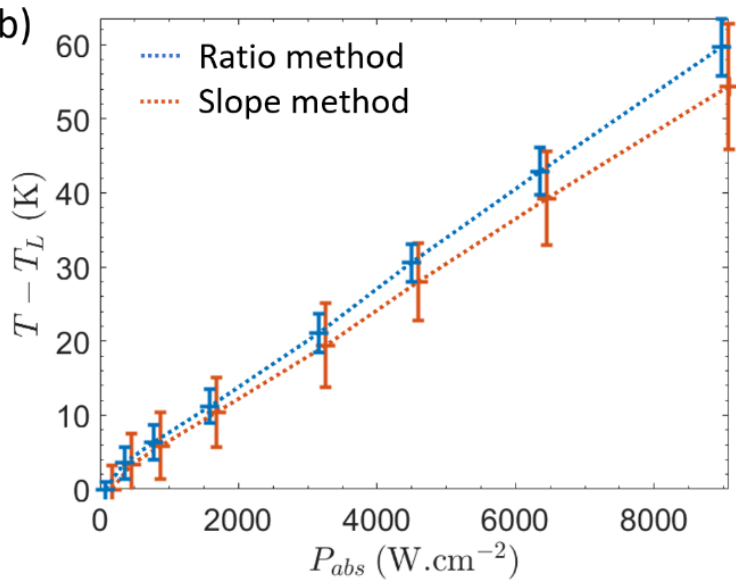

Figure 3: (a) PL ratios $\phi_{n} / \phi_{1}$ in log scale as a function of the photon energies, for the PL data of the $200 \mathrm{~nm}$-thick GaAs absorber with the $532 \mathrm{~nm}$ source, shown in Figure 2. The high-energy slope of these curves can be fitted following Eq. (4), from which we can extract the temperature for all absorbed powers. (b) Carrier temperature increase as a function of absorbed power, for the ratio method (in blue) and the slope method (in red), with error bars. The error on the temperature determination is about twice lower when considering the ratio method. (The points for the slope method are slightly shifted horizontally to make the comparison easier).

\section{RESULTS}

Using the PL ratio method, we determine the carrier temperature increase as a function of the absorbed power for all samples, and both lasers (see Supplementary
Information for the uncertainty calculation for the temperature and the absorbed power). We show the results for both lasers in Figure 4 (except for the $20 \mathrm{~nm}$ absorber with the $638 \mathrm{~nm}$ laser, not shown due to large uncertainties). 

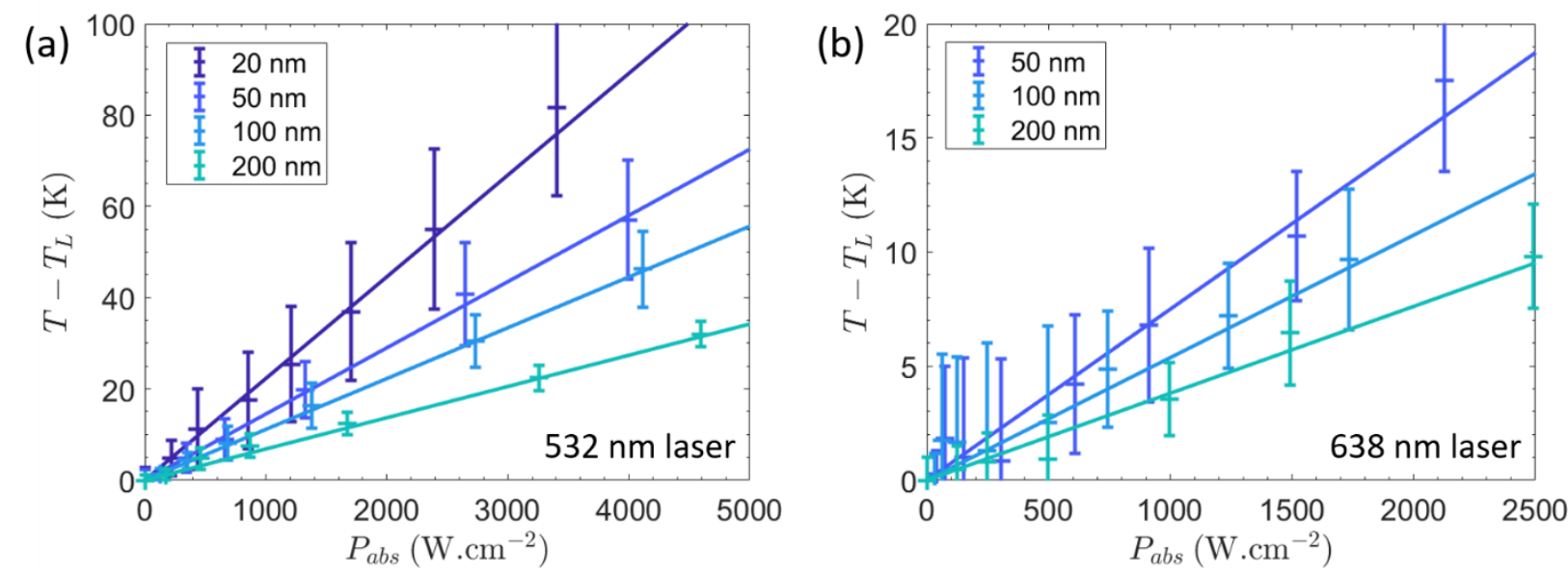

Figure 4: Carrier temperature increase $T-T_{L}$ determined from the ratio method as a function of the absorbed power $P_{a b s}$, for the different GaAs absorbers thicknesses (a) for a $532 \mathrm{~nm}$ laser illumination. (b) for a $638 \mathrm{~nm}$ laser illumination. For all wavelengths and thicknesses, we observe a proportionality between both quantities.

The first thing to be noted is that the temperature increase is relatively modest, always below $100 \mathrm{~K}$ in all samples, within the power range considered. Therefore, we are at the onset of the generation of hot carriers. Because the power range is higher with our $532 \mathrm{~nm}$ laser, the temperature increase with the $638 \mathrm{~nm}$ laser is even more limited, down to about $20 \mathrm{~K}$. Still, because the error is small, we can confirm that for each sample and laser wavelength, we observe hot carriers. To the best of our knowledge, this is the first time that hot carriers have been measured after transferring the epitaxial layer onto a host substrate, an important step for the effective utilization of hot carriers in devices.

A remarkable feature of all the measurements in Figure 4 is that the inferred carrier temperature increases proportionally with the absorbed power $P_{a b s}$. We can translate this observation into a linear relationship

$$
P_{a b s}=Q_{a b s}\left(T-T_{L}\right)
$$

where $T$ is the carrier temperature, $T_{L}$ is the lattice temperature, and $Q_{a b s}$ (in W. $\mathrm{cm}^{-2} \cdot \mathrm{K}^{-1}$ ) is the proportionality coefficient between power and temperature increase. The factor $Q_{a b s}$ is analogous to the thermalization coefficient $Q$ defined by Le Bris et al. ${ }^{33}$, who first observed similar linearity. In the discussion, we will justify why we modified the relationship between thermalized power and carrier temperature increase compared to that reference. To get a better hot-carrier absorber, $Q_{a b s}$ should be as small as possible so that we can generate hot carriers with relatively low incident power. We extract a coefficient $Q_{a b s}$ for every sample and laser wavelength (Figure 5).

This coefficient depends on the absorber thickness and decreases with the absorber thickness: for the same absorbed power, the carrier temperature is higher in thinner absorbers (Figure 5). This result agrees with the general argument made by Le Bris et al. that the thermalization rate should decrease in thinner absorbers ${ }^{23}$. Another key observation is that $Q_{a b s}$ depends on the laser wavelength: it is higher for longer wavelengths. Part of the reason for this will be explained in the discussion, section 4.1, which will lead to an improved definition of the thermalization coefficient $Q$.

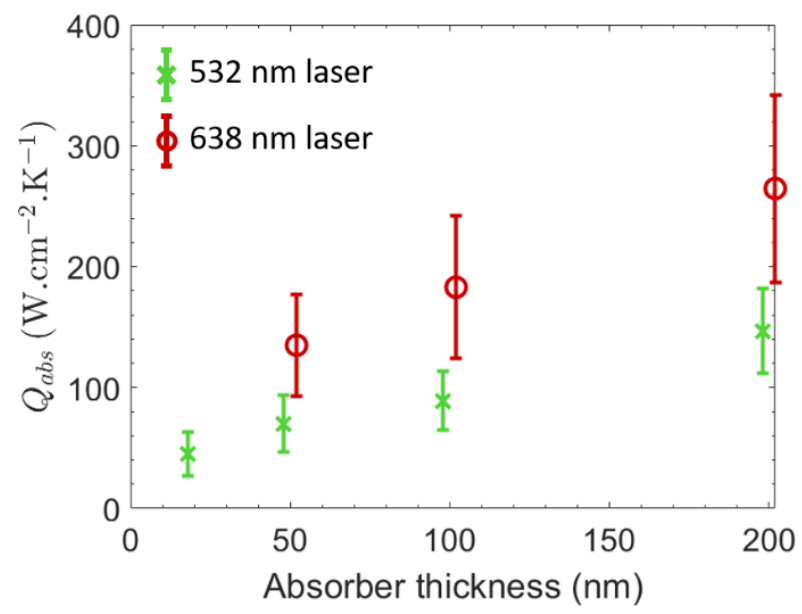

Figure 5: Proportionality coefficient $Q_{a b s}$ as a function of the absorber thickness for both laser wavelengths. $Q_{a b s}$ increases as the absorber thickness increases and is higher for a longer laser 
wavelength. The data for both lasers are slightly shifted along the horizontal axis to improve visibility.

\section{DISCUSSION}

\subsection{Relation between absorbed and thermalized power}

In the previous section, we observed a linear evolution of the carrier temperature increase with the absorbed power $P_{a b s}$. To analyze the relationship between thermalized power $P_{t h}$ and carrier temperature, we first need to express $P_{t h}$ as a function of $P_{a b s}$. Le Bris et al. previously assumed ${ }^{33}$, for simplicity, that all the absorbed power should be thermalized $\left(P_{t h} \approx P_{a b s}\right)$. but we would like to revisit this hypothesis.

The thermalized power is the amount of power dissipated by photo-generated electrons and holes before they recombine. "Because interband recombination rates (both radiative and non-radiative) tend to be much slower (typically in nanoseconds) than electron-phonon scattering rates (typically in picoseconds), the processes of thermalization and recombination can be considered independently." The absorbed power is dissipated entirely through a combination of interband recombinations $P_{\text {inter }}$ and thermalized power $P_{t h}$, leading to the detailed balance equation

$$
P_{a b s}=P_{\text {inter }}+P_{t h}
$$

Under a laser illumination, all electron-hole pairs are generated with the same energy $E_{\text {laser }}$. Let us call the average energy dissipated through interband recombinations $\left\langle E_{\text {inter }}\right\rangle . P_{t h}$ can be expressed as a function of the absorbed power:

$$
P_{t h}=\frac{E_{\text {laser }}-\left\langle E_{\text {inter }}\right\rangle}{E_{\text {laser }}} P_{a b s}
$$

This model assumes that the Auger effect and impact ionization are negligible and that the mechanisms related to interband recombinations are independent of those of thermalization. These interband recombinations can be divided between radiative and non-radiative recombinations.

On one hand, because hot electrons and holes each have an average kinetic energy of $\frac{3}{2} k_{B} T$ (3D system), the average energy of non-radiative recombinations should be

$$
\left\langle E_{\text {inter }}\right\rangle_{\text {nrad }} \approx E_{G}+3 k_{B} T,
$$

where $E_{G}=1.424 \mathrm{eV}$ is the bandgap of $\mathrm{GaAs}$ at room temperature.

On the other hand, the average energy of radiatively recombined photons, assuming step absorption and in the Boltzmann approximation, is

$$
\left\langle E_{\text {inter }}\right\rangle_{\text {rad }} \approx E_{G}+k_{B} T .
$$

This value is different from Eq. (8) because of momentum conservation (not every electron can recombine radiatively with every hole). Here, we will assume that nonradiative recombinations dominate, as is typically the case, and thus consider Eq. (8). Moreover, carrier heating is relatively low in our experiments. Therefore, we can consider $T \approx T_{L}$ in Eq. (8) to obtain a proportionality between $P_{t h}$ and $P_{a b s}$, independent (to a first-order approximation in $T-T_{L}$ ) of the carrier temperature:

$$
P_{\text {th }} \approx \frac{E_{\text {laser }}-\left(E_{G}+3 k_{B} T_{L}\right)}{E_{\text {laser }}} P_{a b s} .
$$

From Eqs. (5) and (10), we define a thermalization coefficient

$$
Q=\frac{E_{\text {laser }}-\left(E_{G}+3 k_{B} T_{L}\right)}{E_{\text {laser }}} Q_{a b s}
$$

such that

$$
P_{t h}=Q\left(T-T_{L}\right)
$$

$Q$ is independent of the carrier temperature, at least for a small temperature increase. The thermalization coefficients obtained for both lasers are shown in Figure 6 . The ratio between the values obtained for both lasers is

$$
\frac{Q(638)}{Q(532)}=1.23 \pm 0.52
$$




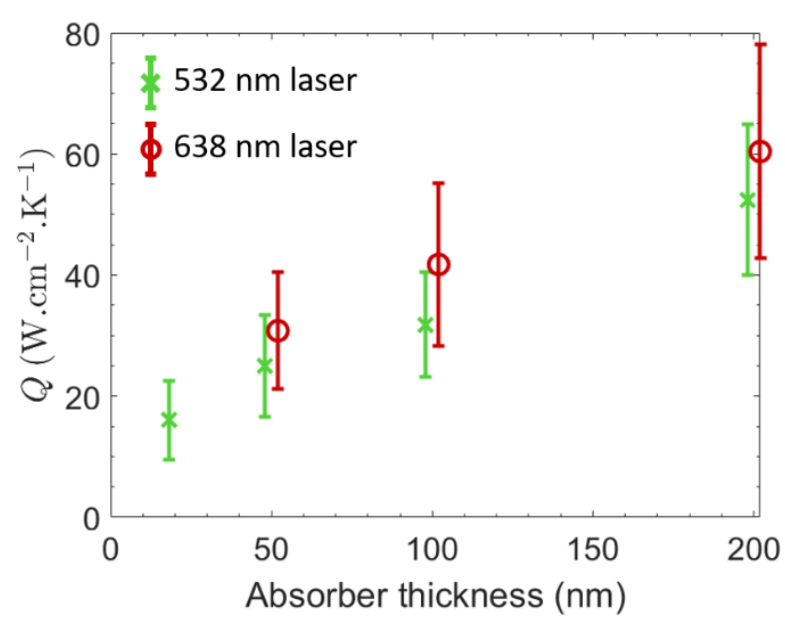

Figure 6: Thermalization coefficient $Q$ as a function of the absorber thickness for both laser wavelengths. $Q$ increases as the absorber thickness increases and is slightly higher for a longer laser wavelength (within error bars). The data for both lasers are slightly shifted along the horizontal axis to improve visibility.

The thermalization coefficients are similar, slightly higher for the lower laser energy (with a large error bar). We will suggest a possible reason for this in the following sections.

\subsection{Thermalization coefficient as a function of absorber thickness}

In the previous section, we gave a broad definition of thermalization (all the power dissipation which is not related to interband recombinations). Now, we want to identify the possible mechanisms for thermalization, i.e. how the extra energy initially given to the carriers is dissipated. In GaAs, as mentioned in the introduction, the carriers interact mostly with the LO phonons, in an elastic manner, such that an equilibrium can be considered between the two populations. Therefore, thermalization corresponds to either high-energy electrons or LO phonons being dissipated from where they are generated, which can take 3 different forms (Figure 7). The first is the decay of optical phonons within the volume where carriers are generated, corresponding to a power dissipated per unit of volume $p_{t h, V}$. The second is the escape of either high-energy carriers or LO phonon through the interface, or their decay at the interface, corresponding to a power dissipated per unit of surface $P_{t h, S}$. The last is the escape of either electrons or LO phonons laterally outside of the incident spot, where they then decay, corresponding to a power dissipated $P_{\text {th,lat }}$. The LO phonons being generated close to the center of the Brillouin zone (because the photocarriers are located there), their wave vector is very short, and their lateral diffusion is negligible relative to the spot size. Moreover, the area from which we measure a PL signal is roughly the same as the laser spot size (see Supplementary Information), indicating no significant lateral carrier diffusion. Therefore, we will assume that lateral thermalization can be neglected.

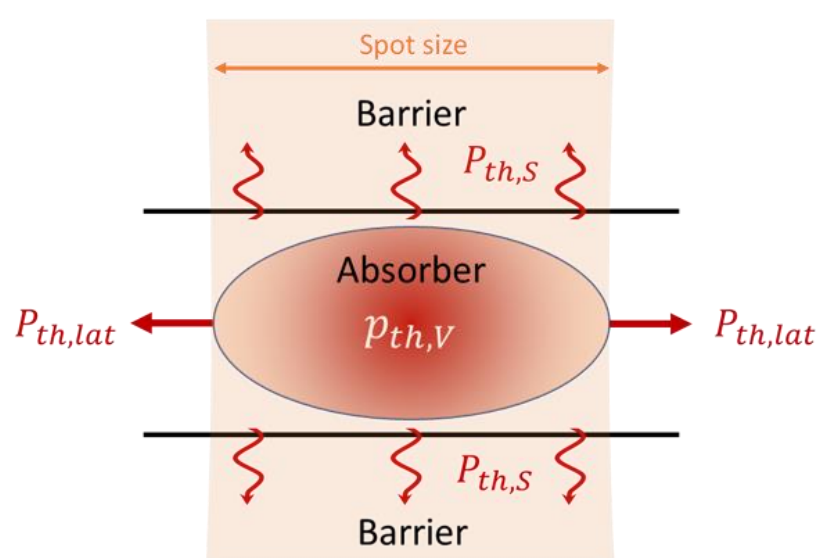

Figure 7: The power which is thermalized in the absorber can follow three paths. The first is thermalization within the volume of the absorber $p_{t h, V}$, which in GaAs is understood to originate mostly from LO phonon decay. The second is thermalization through (or at) the interfaces $P_{t h, S}$, which is for example the case of thermionic emission. The last is the lateral diffusion of either electrons or LO phonons $P_{t h \text {,lat }}$ away from the laser spot, which we consider negligible.

We now look at how the thermalization coefficient varies with the absorber thickness (Figure 8). We observe for both laser wavelengths an affine relationship between thermalization coefficient and thickness, which does not go through the origin. We interpret this by writing

$$
Q=d q_{V}+2 Q_{S}
$$

where $q_{V}$ (in W. $\mathrm{cm}^{-2} \cdot \mathrm{K}^{-1} \cdot \mathrm{nm}^{-1}$ ) is the volume thermalization coefficient and $Q_{S}$ (in $\mathrm{W} . \mathrm{cm}^{-2} \cdot \mathrm{K}^{-1}$ ) is the surface thermalization coefficient of the sample series. 

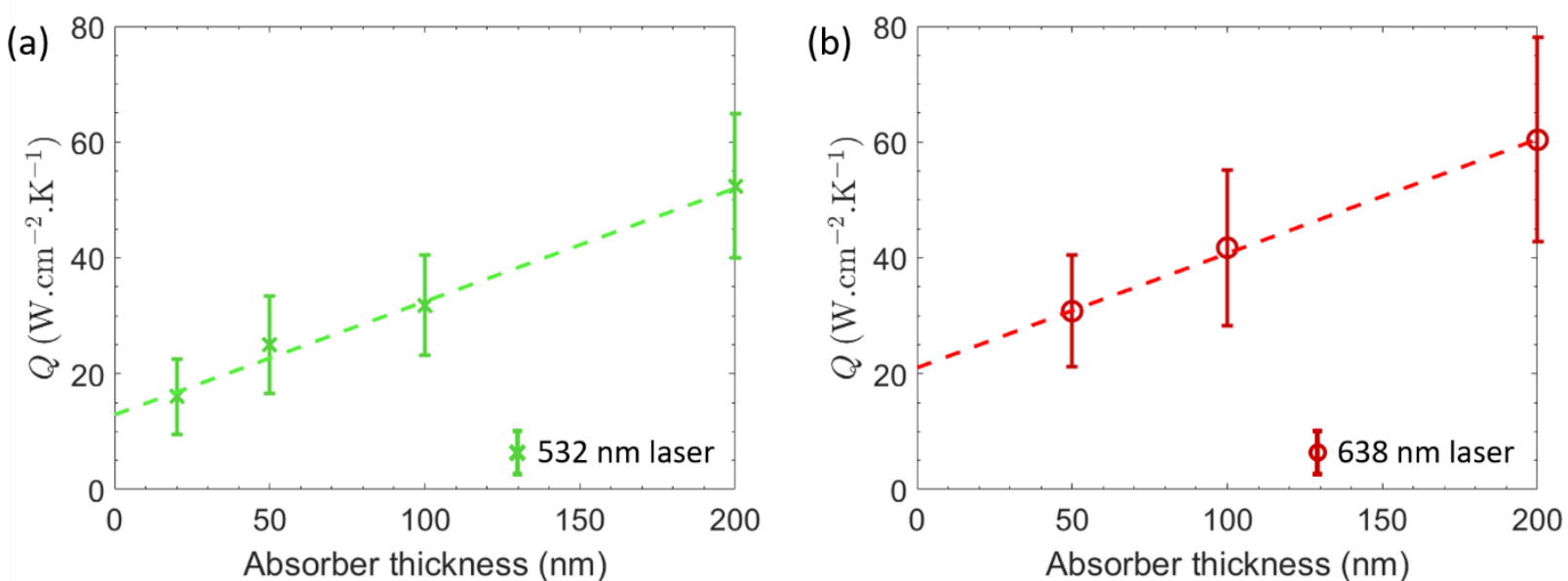

Figure 8: Thermalization coefficient $Q$ as a function of the absorber thickness. (a) For the $532 \mathrm{~nm}$ laser measurement. (b) For the $638 \mathrm{~nm}$ laser measurement. In both cases, we observe an affine relation between both quantities, which allows us to define surface and volume contributions to thermalization following Eq. (14).

Eq. (14) confirms the idea that there are two components to thermalization (Figure 7), such that

$$
P_{t h}=p_{t h, V} \times d+2 P_{t h, S}
$$

The first is proportional to the absorber thickness, related to a thermalization process occurring within the absorber volume, while the second is independent of the thickness and attributable to a thermalization process occurring through the interfaces between the absorber and the barriers. The factor 2 in Eq. (14) is introduced so that $Q_{S}$ corresponds to the thermalization through one GaAsAlGaAs interface, both being assumed identical. Eq. (14) furthermore indicates that both the volume and surface thermalization mechanisms are proportional to the carrier temperature increase, at least up to $T-T_{L}=100 \mathrm{~K}$. Experimental values for these thermalization coefficients for both laser wavelengths are reported in Table I.

\begin{tabular}{|c|c|c|}
\cline { 2 - 3 } \multicolumn{1}{c|}{} & $\mathbf{5 3 2} \mathbf{~ n m}$ laser & $\mathbf{6 3 8} \mathbf{~ n m}$ laser \\
\hline $\boldsymbol{q}_{\boldsymbol{V}}\left(\mathrm{W} \cdot \mathrm{cm}^{-2} \cdot \mathrm{K}^{-1} \cdot \mathrm{nm}^{-1}\right)$ & $0.20 \pm 0.07$ & $0.20 \pm 0.13$ \\
\hline $\boldsymbol{Q}_{S}\left(\mathrm{~W} \cdot \mathrm{cm}^{-2} \cdot \mathrm{K}^{-1}\right)$ & $6.4 \pm 3.2$ & $10.8 \pm 6.9$ \\
\hline
\end{tabular}

Table I: Volume and surface thermalization coefficients obtained from analyzing the variations of the thermalization coefficient with the absorber thickness (Eq. (14)) in the GaAs sample series, for both laser wavelengths.

Very interestingly, we obtained the same volume thermalization coefficient for both lasers. Regarding the surface thermalization coefficient, it appears to be slightly higher with the $638 \mathrm{~nm}$ laser, although the uncertainty is large. The higher thermalization coefficient observed for the
$638 \mathrm{~nm}$ laser (Figure 6) thus seems to originate purely from a larger thermalization at the interfaces.

For this sample series, the contribution of $q_{V}$ and $Q_{S}$ is comparable for absorber thicknesses between 30 and $50 \mathrm{~nm}$. Thicker absorbers are dominated by volume thermalization, while thinner ones mostly dissipate the power through surface thermalization. Ultrathin absorbers may therefore show high thermalization rates if the surface contribution is large.

We gave some examples of volume and surface thermalization mechanisms in the introduction. Thermalization through LO phonon decay should be the dominating thermalization mechanism within the absorber volume. Thermionic emission is a typical case of surface thermalization, where the high energy electrons escape through the barriers, decreasing the average energy of the carrier in the absorber. LO phonon transmission through the interface is another possible source of surface thermalization. In the rest of the discussion, we will look at several of these mechanisms, to see if they can explain the linear relation between thermalized power and temperature increase and if the experimental values are compatible with theoretical models.

\subsection{Origin of volume thermalization}

In this section, we show that the volume thermalization component is consistent with LO phonon decay. We assume that electrons interact only with the LO phonons, as is commonly considered in $\mathrm{GaAs}^{34}$. The thermalized power density $p_{t h}$ (in $\mathrm{W} . \mathrm{cm}^{-3}$ ) is the integral over the wave vectors $\boldsymbol{q}$ of the LO phonon population that decays into acoustic phonons per unit of time, times the LO phonon energy: 


$$
p_{t h, V}=\frac{n_{0}}{2} \times \frac{1}{V_{B}} \iiint \mathrm{d}^{3} \boldsymbol{q} \frac{\partial f}{\partial t}(\boldsymbol{q}) E_{L O}(\boldsymbol{q}),
$$

where $n_{0}$ is the density of atoms in GaAs (in $\mathrm{cm}^{-3}$ ), $V_{B}$ is the volume of the first Brillouin zone, $f$ is the occupation number at wave vector $\boldsymbol{q}$, and $E_{L O}$ is the energy of the wave vector. The factor $1 / 2$ arises because there is 1 LO phonon branch in the first Brillouin zone of a fcc lattice for 2 atoms in the real-space Wigner-Seitz cell ${ }^{35}$. The integral runs over the first Brillouin zone, such that $\iiint d^{3} \boldsymbol{q}=V_{B}$. For a fcc lattice, the volume of the Brillouin zone is

$$
V_{B}=4 \times\left(\frac{2 \pi}{a}\right)^{3}
$$

where $a$ is the lattice parameter of GaAs. We define the wave vector module $\left|\boldsymbol{q}_{B}\right|=\frac{2 \pi}{a}$ such that $V_{B}=4 \times\left|\boldsymbol{q}_{B}\right|^{3}$.

In the relaxation time approximation, we can write ${ }^{34}$

$$
\frac{\partial f}{\partial t}(\boldsymbol{q})=\frac{f(\boldsymbol{q})-f_{e q}(\boldsymbol{q})}{\tau(\boldsymbol{q})}
$$

where $\tau$ is the lifetime of the phonons, $f$ is the nonequilibrium phonon density and $f_{e q}$ is the Bose-Einstein LO phonon distribution at lattice temperature, independent of the wave vector:

$$
f_{e q}=f_{B E}\left(T_{L}\right)=\frac{1}{\exp \left(\frac{E_{L O}}{k_{B} T_{L}}\right)-1} .
$$

Because only the phonons with a low wave vector can couple with the electrons, we consider the Einstein model where the phonon energy is independent of its wave vector. For simplicity, we also assume that all phonons have the same lifetime ${ }^{36} \tau(\boldsymbol{q})=\tau_{L O}$, such that

$$
p_{t h, V}=\frac{n_{0}}{2} \frac{E_{L O}}{\tau_{L O}} \times \frac{1}{V_{B}} \iiint \mathrm{d}^{3} \boldsymbol{q}\left[f(\boldsymbol{q})-f_{e q}\right] .
$$

Not all LO phonons couple with photocarriers. LO phonons with a large wave vector cannot be generated during hot-carrier thermalization and thus remain at equilibrium. We consider as an approximation that only the LO phonons with a wave vector with a module lower than a certain value $\left|\boldsymbol{q}_{\text {max }}\right|$ are coupled with the electrons. We also assume that these phonons are at the same temperature as the electrons. There is also a minimum wave vector $\left|\boldsymbol{q}_{\min }\right|$ such that phonons with a small wave vector are also not coupled with the electrons, but it can be neglected because $\left|\boldsymbol{q}_{\text {min }}\right|^{3} \ll\left|\boldsymbol{q}_{\text {max }}\right|^{3}$. The phonon number then follows one of two Bose-Einstein distributions:

$$
f(\boldsymbol{q})=\left\{\begin{array}{c}
f_{B E}(T) \text { if }|\boldsymbol{q}|<\left|\boldsymbol{q}_{\max }\right| \\
f_{B E}\left(T_{L}\right) \text { otherwise }
\end{array} .\right.
$$

The thermalized power density is then obtained by developing the integral in Eq. (20) in spherical coordinates, leading to

$$
p_{t h, V}=\frac{\pi}{6} \frac{n_{0} E_{L O}}{\tau_{L O}}\left[f_{B E}(T)-f_{B E}\left(T_{L}\right)\right]\left(\frac{\left|\boldsymbol{q}_{\max }\right|}{\left|\boldsymbol{q}_{\boldsymbol{B}}\right|}\right)^{3} .
$$

If the temperature of the carriers is much higher than that of the lattice, then we can neglect the second term in the brackets. In the Boltzmann approximation, we then recover the expression derived by Le Bris et al. ${ }^{33}$. For small temperature increases like those we observe (less than $200 \mathrm{~K}$ for a lattice temperature $T_{L}=300 \mathrm{~K}$ ), the variation of phonon occupancy is very well approximated by a firstorder Taylor expansion around $T-T_{L}=\Delta T$ :

$$
f_{B E}(T)-f_{B E}\left(T_{L}\right) \approx \beta \times \Delta T,
$$

with

$$
\beta=\frac{E_{L O}}{k_{B} T_{L}^{2}} \frac{\exp \left(\frac{E_{L O}}{k_{B} T_{L}}\right)}{\left[\exp \left(\frac{E_{L O}}{k_{B} T_{L}}\right)-1\right]^{2}} .
$$

This leads to the expression

$$
p_{t h, V}=\frac{\pi}{6} \frac{\beta n_{0} E_{L O}}{\tau_{L O}}\left(\frac{\left|\boldsymbol{q}_{\max }\right|}{\left|\boldsymbol{q}_{\boldsymbol{B}}\right|}\right)^{3} \Delta T .
$$

Eq. (25) could explain the linear relationship we observe between thermalized power and temperature increase, provided the other quantities are independent of the temperature. We can express the volume thermalization coefficient

$$
q_{V}=\frac{\pi}{6} \frac{\beta n_{0} E_{L O}}{\tau_{L O}}\left(\frac{\left|\boldsymbol{q}_{\max }\right|}{\left|\boldsymbol{q}_{\boldsymbol{B}}\right|}\right)^{3} .
$$

Most of the parameters in Eq. (26) are well known in GaAs and are independent (at least to a first-order approximation) of the carrier temperature. We consider $n_{0}=4.4210^{22} \mathrm{~cm}^{-3}, E_{L O}=36.110^{-3} \mathrm{eV}, \tau_{L O}=3.5 \mathrm{ps}$ 
and $\left|\boldsymbol{q}_{B}\right|=1.1110^{8} \mathrm{~cm}^{-1}$. The proportionality factor $\beta=$ $2.010^{-3} \mathrm{~K}^{-1}$ is obtained directly from $E_{L O}$ and the lattice temperature $T_{L}=296 \mathrm{~K}$ (Eq. (24)). The main uncertainty comes from the fraction of phonon modes coupled with the electrons, related to the wave vector $\left|\boldsymbol{q}_{\max }\right|$. We can estimate its value using the volume thermalization coefficient $q_{V}$ determined experimentally (Table I):

$$
\left|\boldsymbol{q}_{\max }\right| \approx 3.3 \pm 0.4 \times 10^{6} \mathrm{~cm}^{-1} .
$$

$\left|\boldsymbol{q}_{\text {max }}\right|$ is relatively small compared to $\left|\boldsymbol{q}_{\boldsymbol{B}}\right|$, verifying that only a small fraction of the phonon modes are coupled to the electrons. We want to check whether this order of magnitude for $q_{\max }$ is coherent with the distribution of nonequilibrium phonon densities. We assumed that the phonon occupation number follows the Bose-Einstein distribution at the carrier temperature for the phonon modes coupled with the electrons, while it is equal to the equilibrium distribution for uncoupled modes. In reality, there is no such sharp distinction between coupled and non-coupled modes, and the distribution with $|\boldsymbol{q}|$ should be smoother.

We simulate the non-equilibrium distribution of phonons following the work of $\mathrm{Tsai}^{34}$ (Figure 9). The overall shape of the distribution does not change much as we change the carrier temperature between 300 and $400 \mathrm{~K}$, so we can assume that $\left|\boldsymbol{q}_{\text {max }}\right|$ is independent of the incident power to a first-order approximation. The thermalization coefficient is thus also independent of the laser wavelength, as observed experimentally. The experimental value we determined for $\left|\boldsymbol{q}_{\max }\right|$ agrees with the wave vector at which the modeled non-equilibrium distribution strongly decreases. This meaning LO phonon decay is likely to be responsible for volume thermalization. The precise calculation of the power thermalized in this way requires the knowledge of the chemical potential of the carriers, and will be the object of a future publication.

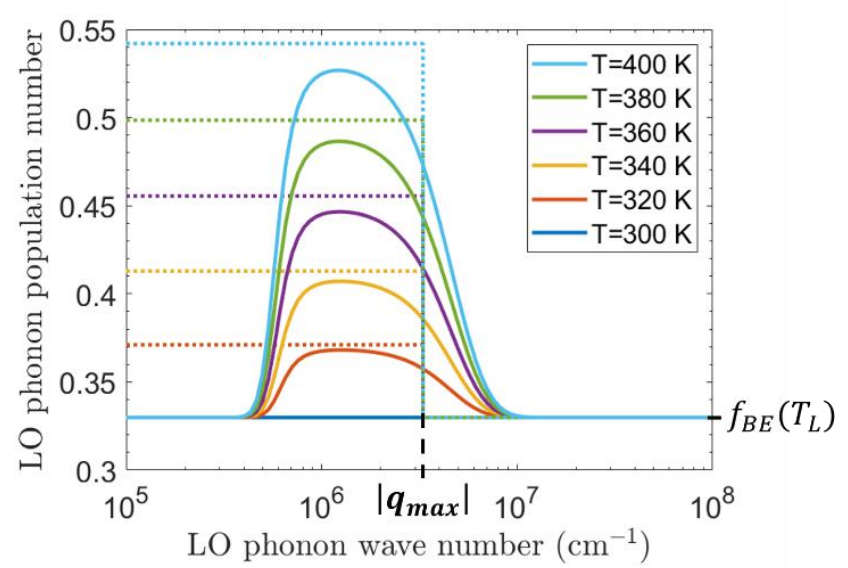

Figure 9: Non-equilibrium LO phonon distribution for a lattice temperature $T_{L}=300 \mathrm{~K}$ and a carrier temperature $T$ varied between 300 and $400 \mathrm{~K}$. The dashed lines are the distributions introduced in Eq. (21): the modes with a wave vector smaller that $\left|\boldsymbol{q}_{\text {max }}\right|$ (determined experimentally in Eq. (27)) follow a Bose-Einstein distribution at the temperature $T$. The continuous lines are the non-equilibrium distributions as calculated from $\mathrm{Tsai}^{34}$ for a Fermi level for the electrons $30 \mathrm{meV}$ below the conduction band. Only the electron contribution to thermalization is considered. We see that the non-equilibrium distributions decrease around the experimentally-derived value of $\left|\boldsymbol{q}_{\text {max }}\right|$, independently from the carrier temperature.

\subsection{Possible origins of surface thermalization}

The surface thermalization coefficient corresponds to energy loss from the electron-LO phonon system, at or through the absorber interfaces. We will show here that it does not originate from the transmission of LO phonons into the barriers.

Because optical phonon dispersion curves are nearly flat close to the zone center, their group speed is small compared to that of acoustic phonons, so that their transport properties are usually neglected. However, since we are concerned only with LO phonons and because our absorbers are very thin, the contribution of LO phonon transmission to hot-carrier thermalization should be assessed. We assume that a fraction $\vartheta(\boldsymbol{q})$ of the incident LO phonons are transmitted through the interface. The power flow of LO phonons through the interface with the barrier is calculated similarly to the volume thermalization through LO phonon decay, where we now need to consider the speed of LO phonons along the direction normal to the interface $v_{z}$. The power which is transmitted to one of the barriers writes

$$
P_{t h, S}=\frac{n_{0}}{2 V_{B}} \iiint \mathrm{d}^{3} \boldsymbol{q} \vartheta(\boldsymbol{q})\left[f(\boldsymbol{q})-f_{e q}(\boldsymbol{q})\right] E_{L O}(\boldsymbol{q}) v_{z}(\boldsymbol{q}),
$$


where the integral runs over the wave vectors in the first Brillouin zone satisfying $v_{z}(\boldsymbol{q}) \geq 0$. Again, we assume that the phonon energy is independent of the wave vector and that phonons with a wave vector of modulus lower than $\left|\boldsymbol{q}_{\text {max }}\right|$ are at temperature $T$, while the others remain cold. We also consider the linear expansion around $\Delta T$ such that

$$
P_{t h, S}=\frac{\beta n_{0} E_{L O}}{2} \times \Delta T \times \frac{1}{V_{B}} \iiint \mathrm{d}^{3} \boldsymbol{q} \vartheta(\boldsymbol{q}) v_{z}(\boldsymbol{q})
$$

where the integral now runs over the half-sphere of radius $\left|\boldsymbol{q}_{\text {max }}\right|$ and $v_{z}(\boldsymbol{q}) \geq 0$. This value admits an upper limit, provided $\vartheta(\boldsymbol{q})=1$ for all wave vectors:

$$
P_{t h, S} \leq \frac{\beta n_{0} E_{L O}}{2} \times \Delta T \times \frac{1}{V_{B}} \iiint \mathrm{d}^{3} \boldsymbol{q} v_{z}(\boldsymbol{q}) .
$$

The integral expression can be developed in spherical coordinates with $v_{z}=v \cos \varphi$, leading to

$$
\iiint \mathrm{d}^{3} \boldsymbol{q} v_{z}(\boldsymbol{q})=\pi \int_{0}^{\left|\boldsymbol{q}_{\max }\right|} \mathrm{d} q q^{2} v(q) .
$$

The LO phonon speed is to a first approximation $(|\boldsymbol{q}| \ll$ $\left.\left|\boldsymbol{q}_{\boldsymbol{B}}\right|\right)$ proportional to the wave vector:

$$
v(q)=\frac{\partial \omega_{L O}}{\partial q} \approx \alpha_{0} \times q
$$

where $\omega_{L O}$ is the frequency of LO phonons. We can get an analytic expression for $\alpha_{0}$ by considering the dispersion relation in the absence of polar interactions:

$$
\alpha_{0}=\frac{E_{L O}}{\hbar}\left(\frac{M_{G a} M_{A s}}{2\left(M_{G a}+M_{A s}\right)^{2}} a^{2}\right)
$$

where $M_{G a}$ and $M_{A s}$ are the atomic masses of Ga and As, respectively, and $a$ is the lattice parameter of GaAs. As mentioned, this description ignores the polar nature of GaAs, which results in separate $\mathrm{LO}$ and TO dispersion relations with phonon energies different from the one derived in Eq. (33) . It is possible to fit $\alpha_{0}$ from experimental or numerical data, but the variety of models and the small curvature mean there is necessarily a lot of uncertainty. We will thus consider the value obtained in Eq. (33) as an order of magnitude for this calculation.

Overall, the upper limit for the power thermalized by LO phonon transport through an interface is

$$
P_{t h, S} \leq \frac{\pi}{32} \alpha_{0} \beta n_{0} E_{L O}\left|\boldsymbol{q}_{\max }\right|\left(\frac{\left|\boldsymbol{q}_{\max }\right|}{\left|\boldsymbol{q}_{\boldsymbol{B}}\right|}\right)^{3} \Delta T
$$

As for the LO phonon decay term, the LO phonon transmission appears to be proportional to the temperature increase $\Delta T$, which stems from the linear expansion of the difference in the occupation functions. The surface thermalization coefficient thus has an upper limit

$$
Q_{S} \leq \frac{\pi}{32} \alpha_{0} \beta n_{0} E_{L O}\left|\boldsymbol{q}_{\max }\right|\left(\frac{\left|\boldsymbol{q}_{\max }\right|}{\left|\boldsymbol{q}_{\boldsymbol{B}}\right|}\right)^{3} .
$$

We consider for $\left|\boldsymbol{q}_{\max }\right|$ the value determined from the experimental volume thermalization coefficient (Eq. (27)), leading to

$$
Q_{S} \leq 9.4 \times 10^{-2} \mathrm{~W} \cdot \mathrm{cm}^{-2} \cdot \mathrm{K}^{-1} .
$$

This value is more than one order of magnitude smaller than the experimental measurements. Moreover, the LO phonon energies of $\mathrm{GaAs}$ and $\mathrm{Al}_{0.5} \mathrm{Ga}_{0.5} \mathrm{As}$ differ significantly ${ }^{37}$, such that the transmission coefficient $\vartheta$ is expected to be much lower than 1 . Therefore, it seems like the contribution of LO phonon transmission to thermalization can be considered negligible here. This important result means that as long as the experimentally measured surface thermalization remains significantly higher than in Eq. (36), there is no need to engineer a phonon mismatch between the absorber and barrier materials.

The principal remaining candidate for surface thermalization is the thermionic emission of electrons over the barriers ${ }^{7,14,38}$. Thermionic emission agrees qualitatively with the observation that the surface thermalization coefficient is higher for the longer laser wavelengths. Indeed, a longer wavelength will lead to more photocarriers generated for the same absorbed power, which should lead to a higher chemical potential in the absorber. Moreover, the $638 \mathrm{~nm}$ laser, contrarily to the $532 \mathrm{~nm}$ laser, does not have enough energy to excite carriers in the barriers, such that the chemical potential in the barriers should be lower for the longer wavelength. Therefore, the thermionic power flux from the absorber to the barriers should be higher with the longer laser wavelength. As a consequence, the thermalization coefficient should increase with the laser wavelength, which is what we observe experimentally. An in-depth analysis of the PL spectra to determine the chemical potentials is required to determine whether thermionic emission is indeed the principal reason for surface thermalization. 
Other possible origins for the thermalization coefficient involve LO phonon or electron scattering events at the interface. These terms are especially difficult to estimate, as they depend on defect states between the absorber and the barrier. What we can say at this stage is that there is significant thermalization through the interfaces in this sample series, especially for ultra-thin absorbers where it dominates the LO phonon decay volume process.

\section{CONCLUSIONS}

We reported power-dependent PL characterization of ultrathin heterostructures with two different laser wavelengths at room temperature. The samples considered are GaAs absorbers of four different thicknesses between 20 and $200 \mathrm{~nm}$ sandwiched between AlGaAs barriers, transferred onto a host substrate. The simplicity of this series provides a way to shed light on hot-carrier thermalization mechanisms and constitutes a step towards the fabrication of more complex hot-carrier solar cell designs.

We considered a PL ratio method to extract the carrier temperature in these ultrathin layers with relatively low uncertainty. We observed a linear relationship between the absorbed power and the temperature increase of the carriers in all samples, enabling the extraction of a proportionality coefficient $Q_{a b s}$. By relating absorbed and thermalized power, we determined a thermalization coefficient $Q$ for all absorber thicknesses and laser wavelengths.

We obtained an affine relationship between the thermalization coefficient and the absorber thickness. From this relationship, we identified two distinct thermalization mechanisms. The first mechanism is a volume effect proportional to the absorber thickness, attributed to LO phonon decay. The second mechanism is a surface contribution independent of the absorber thickness, which highlights the importance of the interfaces in the hot-carrier thermalization. Although its exact origin remains unclear, we confirmed that LO phonon transmission through the barriers is negligible so that there is no need to consider the barriers' LO phonon energy as a design criterion.

Overall, the definition we provided for the thermalization coefficient will be helpful to compare the quality of hot-carrier absorbers across different materials and experimental setups. Furthermore, our approach can be applied to identify surface and volume thermalization contributions in any material. This work also emphasizes the crucial importance of developing ultrathin absorbers as well as the need to consider their interfaces carefully. These ultrathin absorbers could eventually enable high-efficiency hot-carrier solar cells, provided high absorption can be maintained. This absorption enhancement requires the implementation of advanced light trapping schemes, for which promising experimental results were achieved in 25 and 200 nm-thick GaAs layers ${ }^{39,40}$.

Future work includes a more advanced fit of the PL data to extract the chemical potential in the absorber and determine more accurately the contributions of LO phonon decay and thermionic emission. It will also be of interest to investigate quantum confinement of carriers in ultrathin absorbers and its impact on thermalization.

\section{SUPPLEMENTARY MATERIAL}

See supplementary material for a detailed analysis of the error on the determination of the carrier temperature and the thermalization coefficient.

\section{ACKNOWLEDGMENTS}

This work was performed within the framework of LIA NextPV currently IRP NextPV-II, with support from CNRS (INC and INSIS) and RCAST/University of Tokyo. Authors would like to acknowledge the support of the Japanese MEXT scholarship program, the Japan Society for the Promotion of Science (JSPS) KAKENHI Grant Number 19H02541, the French ANR project ICEMAN Grant Number ANR-19-CE05-0019, the IPVF PROOF program, and the Energy4Climate Interdisciplinary Center (E4C) of IP Paris and Ecole des Ponts ParisTech (France).

\section{AIP PUBLISHING DATA SHARING POLICY}

The data that support the findings of this study are available from the corresponding author upon reasonable request.

\section{REFERENCES}

[1] Shockley, W. and Queisser, H. J., "Detailed Balance Limit of Efficiency of $p-n$ Junction Solar Cells," Journal of Applied Physics 32(3), 510-519 (1961).

[2] Ross, R. T. and Nozik, A. J., "Efficiency of hot-carrier solar energy converters," Journal of Applied Physics 53(5), 3813-3818 (1982).

[3] Dimmock, J. A. R., Day, S., Kauer, M., Smith, K. and Heffernan, J., "Demonstration of a hot-carrier photovoltaic cell," Prog. Photovolt: Res. Appl. 22(2), 151160 (2014).

[4] Nguyen, D.-T., Lombez, L., Gibelli, F., Boyer-Richard, S., Le Corre, A., Durand, O. and Guillemoles, J.-F., "Quantitative 
experimental assessment of hot carrier-enhanced solar cells at room temperature," Nature Energy 3(3), 236-242 (2018).

[5] Hirst, L. C., Walters, R. J., Führer, M. F. and Ekins-Daukes, N. J., "Experimental demonstration of hot-carrier photocurrent in an InGaAs quantum well solar cell," Appl. Phys. Lett. 104(23), 231115 (2014).

[6] Jehl, Z., Suchet, D., Miyashita, N., Behagel, B., Giteau, M., Delamarre, A., Jeco, B. M. F. Y., Guillemoles, J. and Okada, Y., "Hot Carrier Extraction Using Energy Selective Contacts and Feedback On The Remaining Distribution," 2018 IEEE 7th World Conference on Photovoltaic Energy Conversion (WCPEC) (A Joint Conference of 45th IEEE PVSC, 28th PVSEC 34th EU PVSEC), 1814-1816 (2018).

[7] Suchet, D., Jehl, Z., Okada, Y. and Guillemoles, J.-F., "Influence of Hot-Carrier Extraction from a Photovoltaic Absorber: An Evaporative Approach," Phys. Rev. Applied 8(3), 034030 (2017).

[8] Conibeer, G. J., Jiang, C.-W., König, D., Shrestha, S., Walsh, T. and Green, M. A., "Selective energy contacts for hot carrier solar cells," Thin Solid Films 516(20), 6968-6973 (2008).

[9] Le bris, A., "Etude de faisabilité d'un dispositif photovoltaïque à porteurs chauds," Ph.D. thesis (2011)

[10] Fröhlich, H., "Electrons in lattice fields," Advances in Physics 3(11), 325-361 (1954).

[11] Klemens, P. G., "Anharmonic Decay of Optical Phonons," Phys. Rev. 148(2), 845-848 (1966).

[12] Shah, J., Pinczuk, A., Gossard, A. C. and Wiegmann, W. "Energy-Loss Rates for Hot Electrons and Holes in GaAs Quantum Wells," Phys. Rev. Lett. 54(18), 2045-2048 (1985).

[13] Hathwar, R., Zou, Y., Jirauschek, C. and Goodnick, S. M., "Nonequilibrium electron and phonon dynamics in advanced concept solar cells," J. Phys. D: Appl. Phys. 52(9), 093001 (2019).

[14] Shakouri, A., LaBounty, C., Piprek, J., Abraham, P. and Bowers, J. E., "Thermionic emission cooling in single barrier heterostructures," Appl. Phys. Lett. 74(1), 88-89 (1998).

[15] Yangui, A., Bescond, M., Yan, T., Nagai, N. and Hirakawa, K., "Evaporative electron cooling in asymmetric double barrier semiconductor heterostructures," 1, Nature Communications 10(1), 4504 (2019).

[16] Behaghel, B., Tamaki, R., Chen, H.-L., Rale, P., Lombez, L. Shoji, Y., Delamarre, A., Cattoni, A., Collin, S., Okada, Y. and Guillemoles, J.-F., "A hot-carrier assisted InAs/AIGaAs quantum-dot intermediate-band solar cell," Semicond. Sci. Technol. 34(8), 084001 (2019).

[17] Conibeer, G., Shrestha, S., Huang, S., Patterson, R., Xia, H., Feng, Y., Zhang, P., Gupta, N., Tayebjee, M., Smyth, S., Liao, Y., Lin, S., Wang, P., Dai, X. and Chung, S., "Hot carrier solar cell absorber prerequisites and candidate material systems," Solar Energy Materials and Solar Cells 135, 124129 (2015).
[18] Pelouch, W. S., Ellingson, R. J., Powers, P. E., Tang, C. L., Szmyd, D. M. and Nozik, A. J., "Comparison of hot-carrier relaxation in quantum wells and bulk GaAs at high carrier densities," Phys. Rev. B 45(3), 1450-1453 (1992).

[19] Rosenwaks, Y., Hanna, M. C., Levi, D. H., Szmyd, D. M., Ahrenkiel, R. K. and Nozik, A. J., "Hot-carrier cooling in GaAs: Quantum wells versus bulk," Phys. Rev. B 48(19), 14675-14678 (1993).

[20] Hirst, L. C., Yakes, M. K., Bailey, C. G., Tischler, J. G., Lumb, M. P., González, M., Führer, M. F., Ekins-Daukes, N. J. and Walters, R. J., "Enhanced Hot-Carrier Effects in InAIAs/InGaAs Quantum Wells," IEEE Journal of Photovoltaics 4(6), 1526-1531 (2014).

[21] Conibeer, G., Zhang, Y., Bremner, S. P. and Shrestha, S., "Towards an understanding of hot carrier cooling mechanisms in multiple quantum wells," Jpn. J. Appl. Phys. 56(9), 091201 (2017).

[22] Zhang, Y., Tang, L., Zhang, B., Wang, P. and Xu, C., "Quantitative study on the mechanisms underlying the phonon bottleneck effect in InN/InGaN multiple quantum wells," Appl. Phys. Lett. 116(10), 103104 (2020).

[23] Bris, A. L., Rodiere, J., Colin, C., Collin, S., Pelouard, J. L., Esteban, R., Laroche, M., Greffet, J. J. and Guillemoles, J. F., "Hot Carrier Solar Cells: Controlling Thermalization in Ultrathin Devices," IEEE Journal of Photovoltaics 2(4), 506-511 (2012).

[24] Achermann, M., Bartko, A. P., Hollingsworth, J. A. and Klimov, V. I., "The effect of Auger heating on intraband carrier relaxation in semiconductor quantum rods," 8 , Nature Phys 2(8), 557-561 (2006).

[25] Strauss, U., Rühle, W. W. and Köhler, K., "Auger recombination in intrinsic GaAs," Appl. Phys. Lett. 62(1), 55-57 (1993).

[26] Giteau, M., Watanabe, K., Miyashita, N., Sodabanlu, H., Atlan, F., Suchet, D., Collin, S., Guillemoles, J.-F. and Okada Y., "Fabrication and optical characterization of ultrathin IIIV transferred heterostructures for hot-carrier absorbers," Physics, Simulation, and Photonic Engineering of Photovoltaic Devices IX 11275, 1127507, International Society for Optics and Photonics (2020).

[27] Knittl, Z., [Optics of thin films: an optical multilayer theory], Wiley, London, New York (1976).

[28] Acquaroli, L. N., "Matrix method for thin film optics," arXiv:1809.07708 [physics] (2018).

[29] Wurfel, P., "The chemical potential of radiation," J. Phys. C: Solid State Phys. 15(18), 3967 (1982).

[30] Gibelli, F., Lombez, L. and Guillemoles, J.-F., "Two carrier temperatures non-equilibrium generalized Planck law for semiconductors," Physica B: Condensed Matter 498, 7-14 (2016).

[31] Whiteside, V. R., Magill, B. A., Lumb, M. P., Esmaielpour, H., Meeker, M. A., Mudiyanselage, R. R. H. H., Messager, A., Vijeyaragunathan, S., Mishima, T. D., Santos, M. B., Vurgaftman, I., Khodaparast, G. A. and Sellers, I. R., "Valence band states in an InAs/AIAsSb multi-quantum 
well hot carrier absorber," Semicond. Sci. Technol. 34(2), 025005 (2019).

[32] Esmaielpour, H., Suchet, D., Lombez, L., Giteau, M., Delamarre, A., Ory, D., Cattoni, A., Collin, S. and Guillemoles, J.-F., "Investigation of hot carrier spatial distributions in quantum well structures via hyperspectral luminescence imaging," J. Appl. Phys. (in press).

[33] Bris, A. L., Lombez, L., Laribi, S., Boissier, G., Christol, P. and Guillemoles, J.-F., "Thermalisation rate study of GaSbbased heterostructures by continuous wave photoluminescence and their potential as hot carrier solar cell absorbers," Energy \& Environmental Science 5(3), 6225-6232 (2012).

[34] Tsai, C.-Y., "Theoretical model and simulation of carrier heating with effects of nonequilibrium hot phonons in semiconductor photovoltaic devices," Progress in Photovoltaics: Research and Applications 26(10), 808-824 (2018).

[35] Kittel, C., [Introduction to Solid State Physics, 8 edition], Wiley, Hoboken, NJ (2004).
[36] Bhatt, A. R., Kim, K. W. and Stroscio, M. A., "Theoretical calculation of longitudinal-optical-phonon lifetime in GaAs," Journal of Applied Physics 76(6), 3905-3907 (1994).

[37] Adachi, S., [Properties of aluminium gallium arsenide], IEE, INSPEC, London (1993).

[38] Könemann, F., Chen, I.-J., Lehmann, S., Thelander, C. and Gotsmann, B., "Imaging the Thermalization of Hot Carriers After Thermionic Emission Over a Polytype Barrier," Phys. Rev. Applied 13(5), 054035 (2020).

[39] Massiot, I., Vandamme, N., Bardou, N., Dupuis, C., Lemaître, A., Guillemoles, J.-F. and Collin, S., "Metal Nanogrid for Broadband Multiresonant Light-Harvesting in Ultrathin GaAs Layers," ACS Photonics 1(9), 878-884 (2014).

[40] Chen, H.-L., Cattoni, A., Lépinau, R. D., Walker, A. W., Höhn, O., Lackner, D., Siefer, G., Faustini, M., Vandamme, N., Goffard, J., Behaghel, B., Dupuis, C., Bardou, N., Dimroth, F. and Collin, S., "A 19.9\%-efficient ultrathin solar cell based on a 205-nm-thick GaAs absorber and a silver nanostructured back mirror," Nat Energy, 1-7 (2019). 
(a) $\mathrm{Al}_{0.5} \mathrm{Ga}_{0.5} \mathrm{As}$ barrier $90 \mathrm{~nm}$

GaAs Absorber 20-200nm

$\mathrm{Al}_{0.5} \mathrm{Ga}_{0.5} \mathrm{As}$ barrier $90 \mathrm{~nm}$

Au mirror

Si support (b) $\mathrm{CB}$

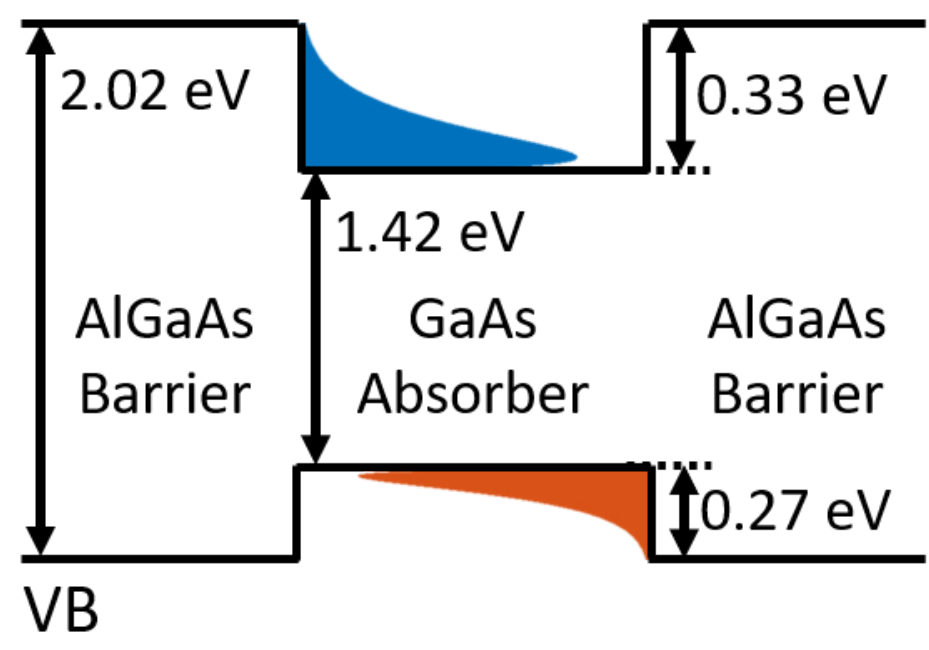




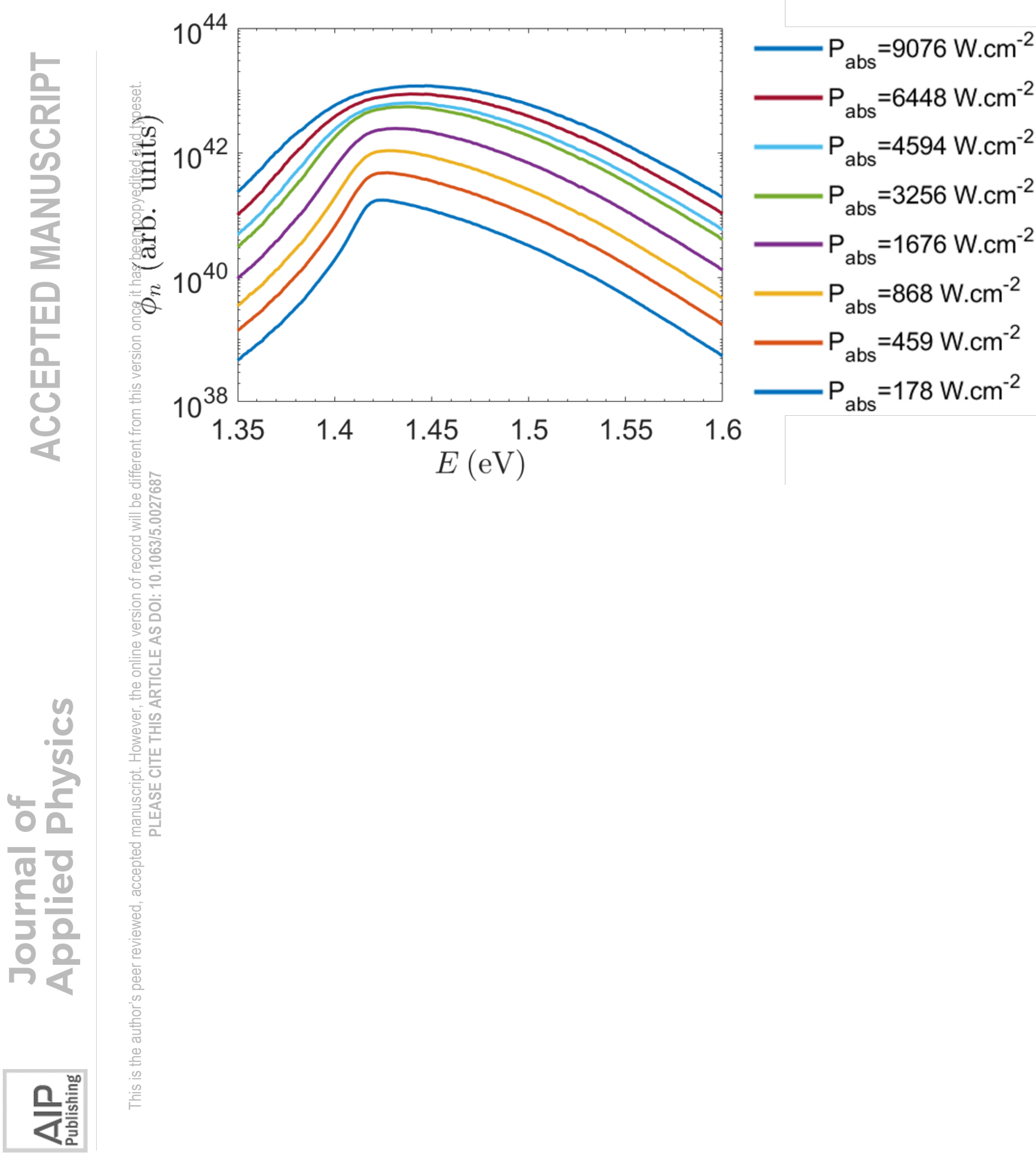



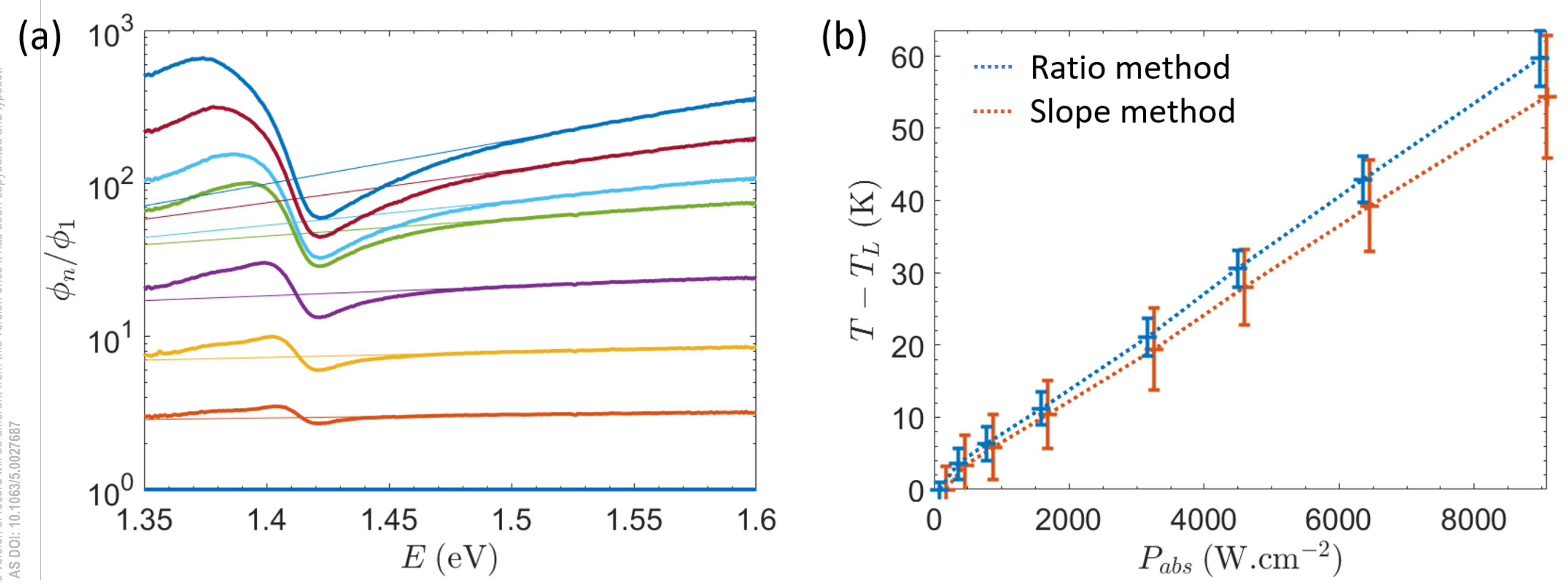

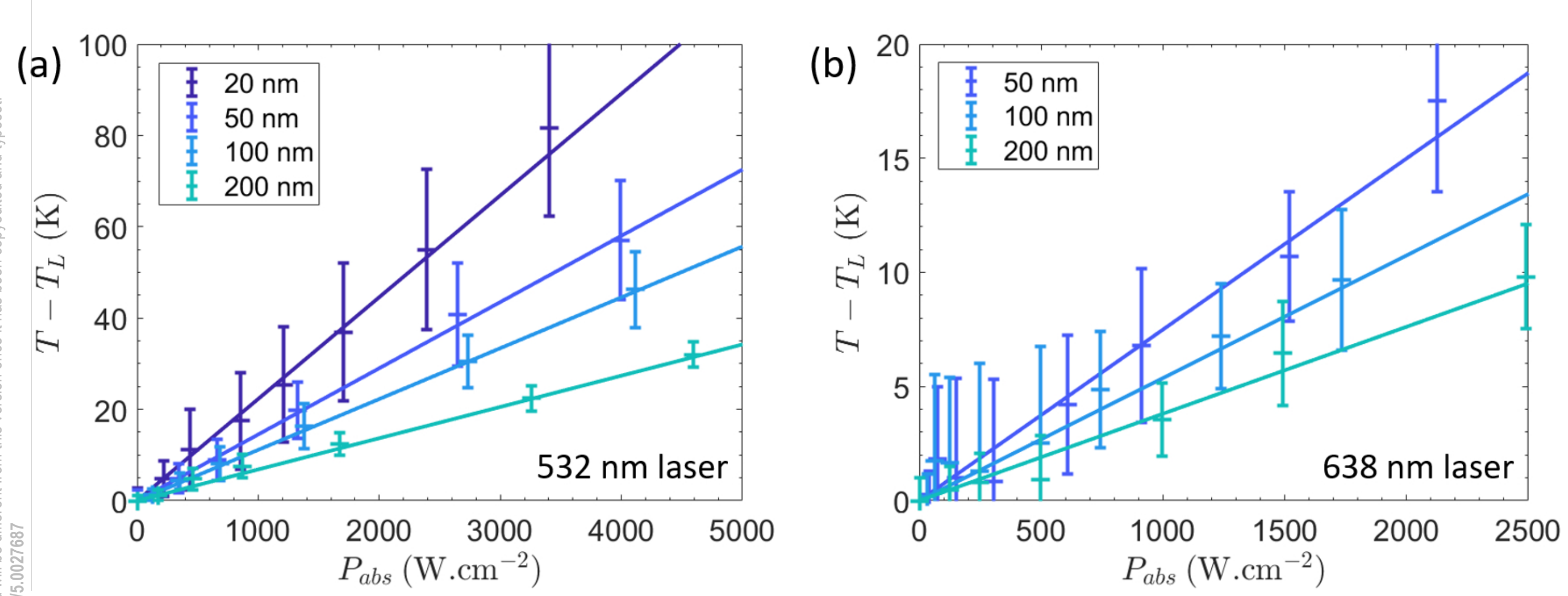


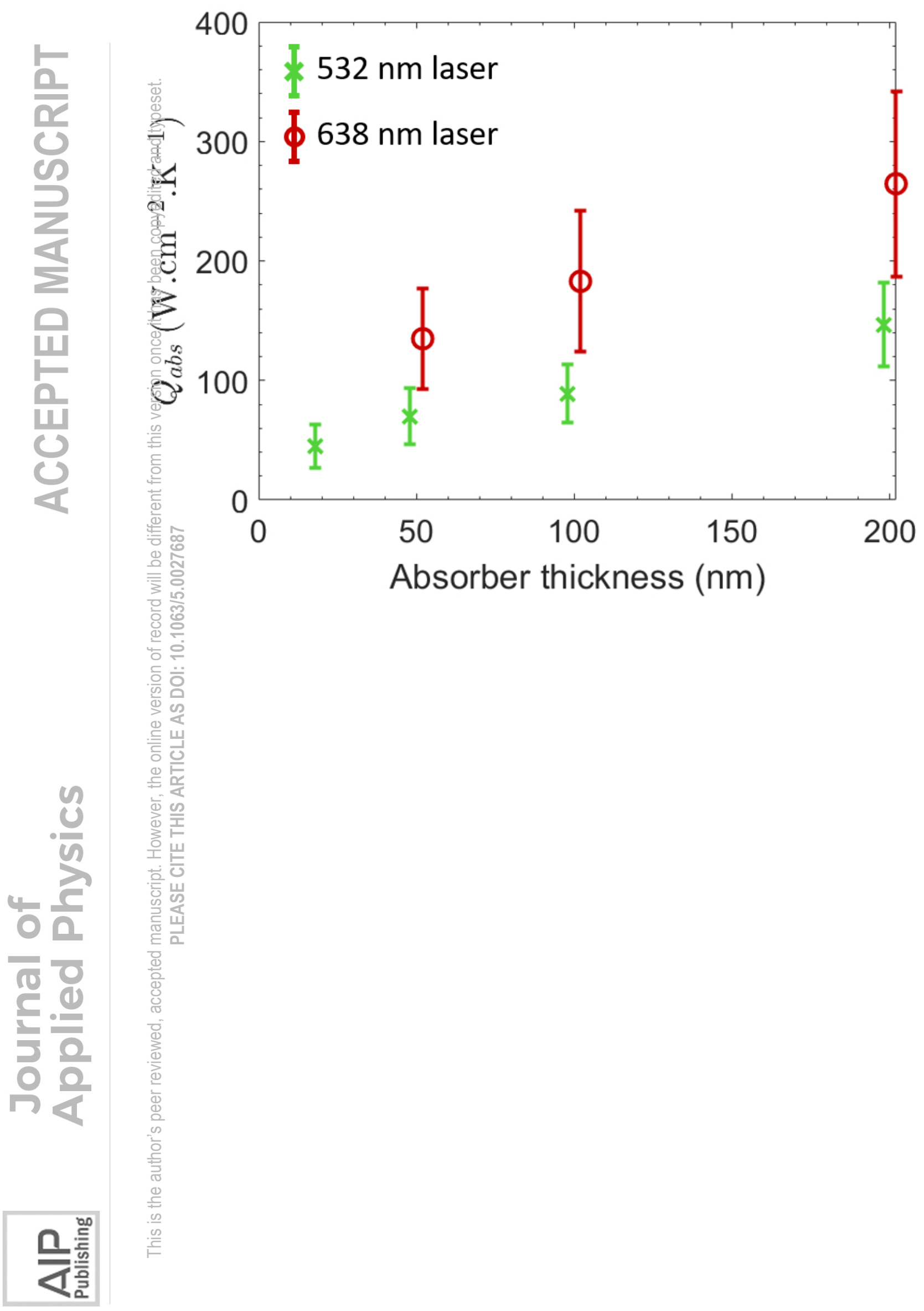




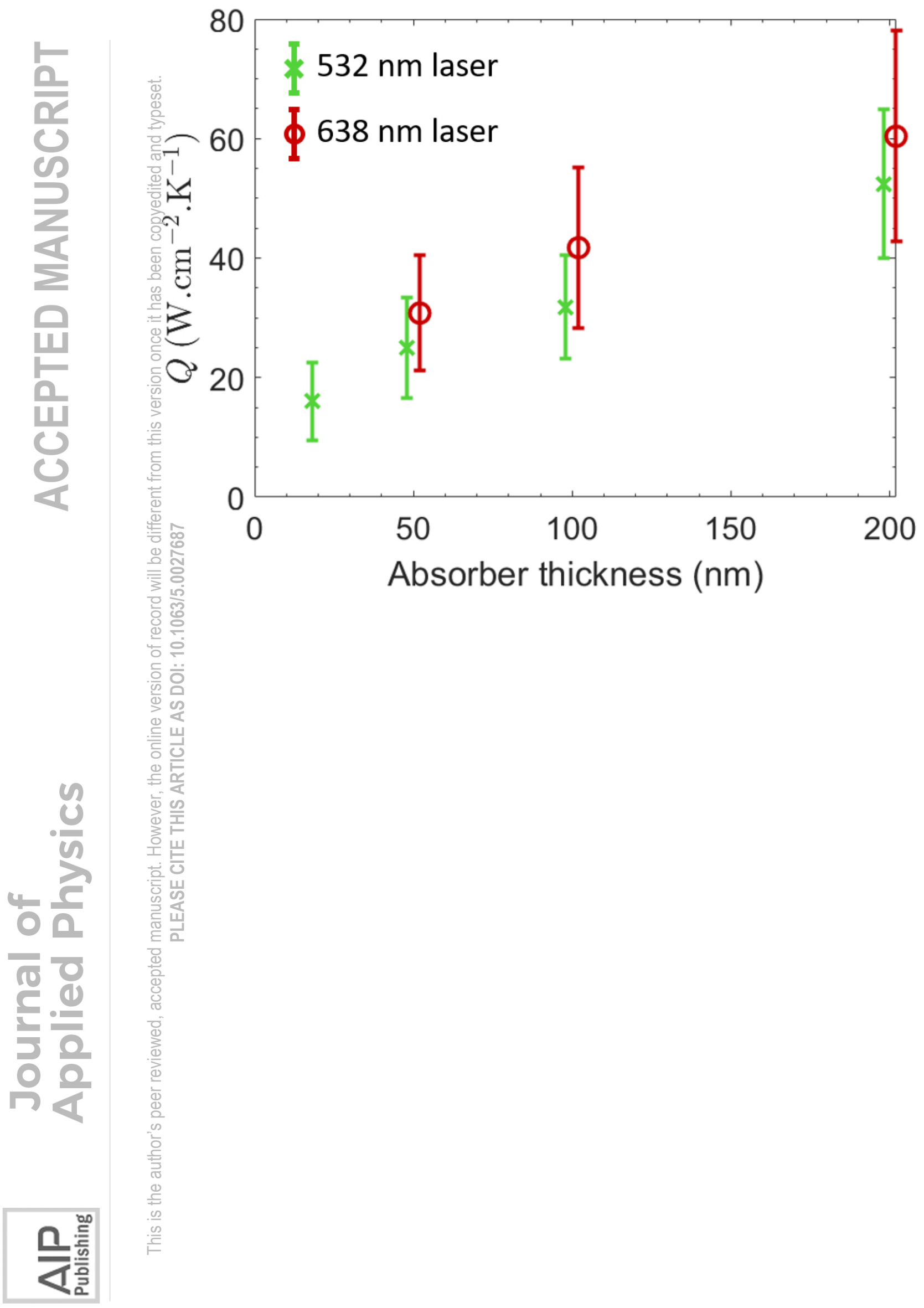


Spot size

\section{Barrier}
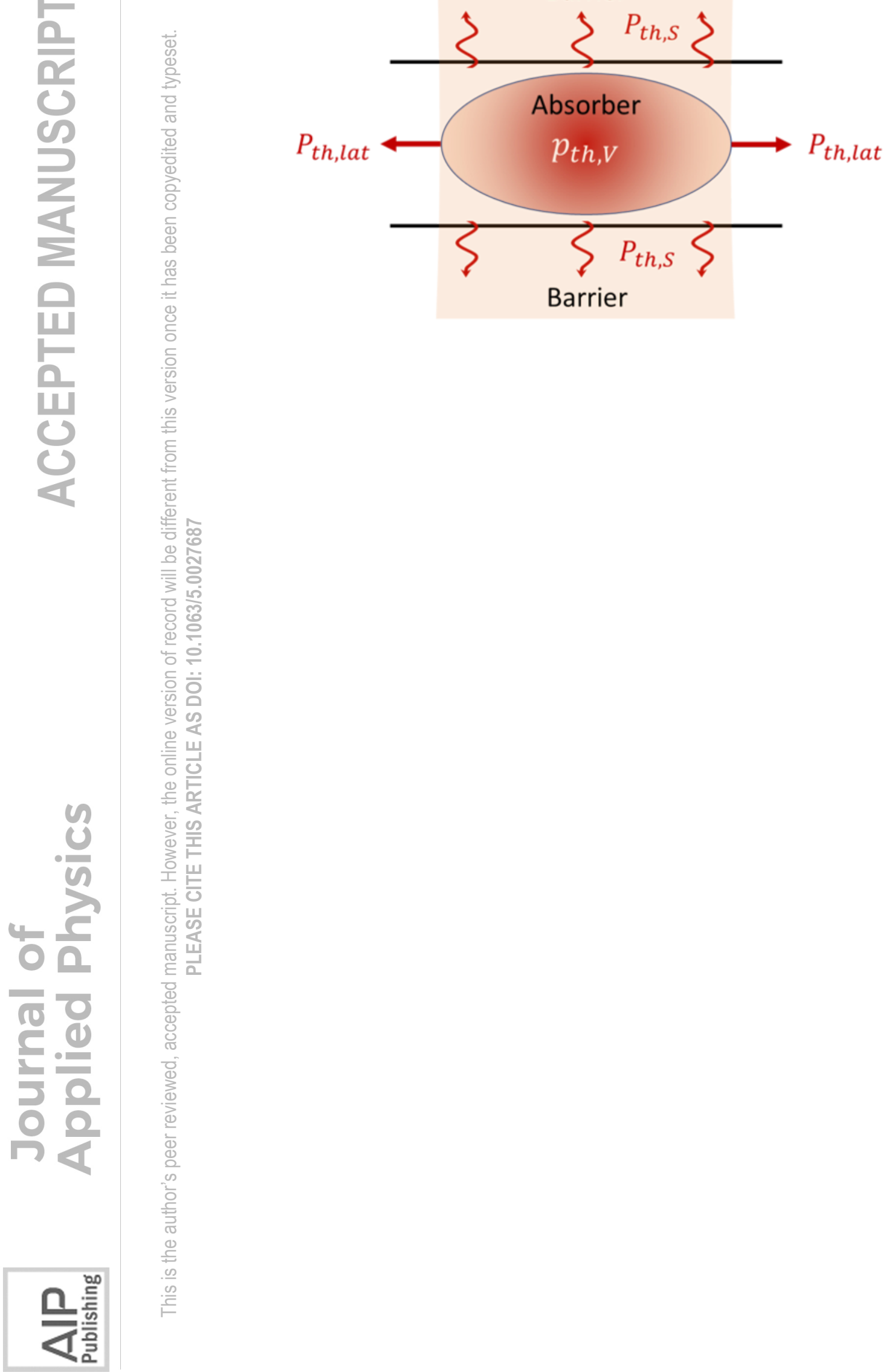


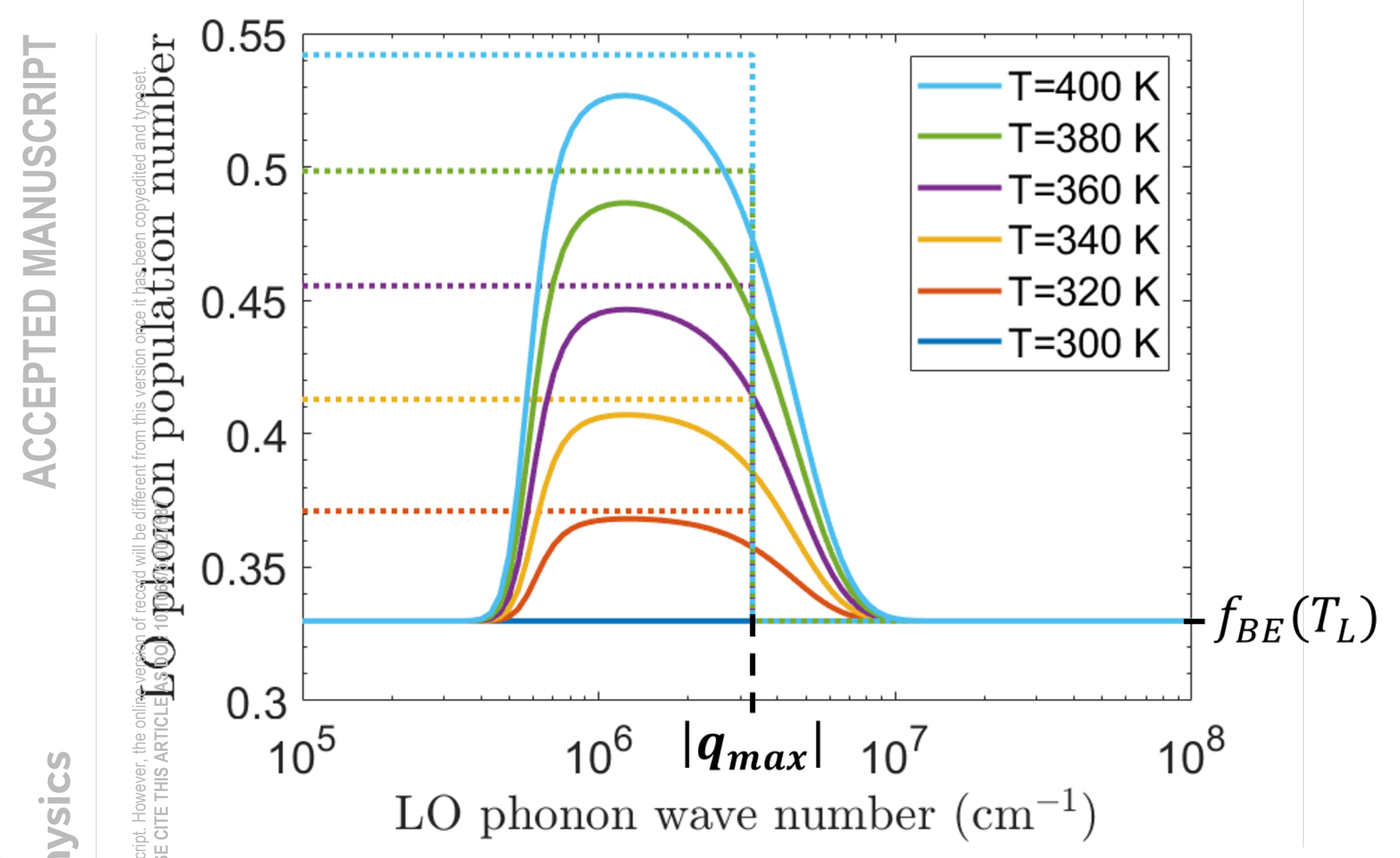

